\title{
Energia de activação? Qual?
}

\section{Introdução}

Todo o cinético - todo o químico - afirmará, sem qualquer relutância: «É fundamental conhecer o significado de Energia de Activação».

Mas o que é Energia de Activação?

Aqui, um aluno, se interpelado, titubeará - e, provavelmente, também o professor.

Uma primeira dificuldade na resposta resulta de, ao termo genérico Energia de Activação, corresponderem vários conceitos, muitas vezes inter-relacionados mas numericamente diferentes, como sejam a Energia de Activação de Arrhenius, $\mathrm{E}_{\mathrm{a}}$, a Barreira de Energia Potencial, $\mathrm{V}_{\mathrm{B}}$, a Energia (Interna) de Activação do Ponto Zero, $\Delta^{\neq} \mathrm{U}_{0}$, a Energia Interna de Activação, $\Delta^{\ddagger} \mathrm{H}_{\mathrm{T}}$, e a Entalpia de Activação, $\Delta^{\neq} \hat{\mathbf{H}}_{\mathrm{T}}$, entre outras; uma segunda, surge, ainda, na própria interpretação de cada um destes conceitos ${ }^{1}$.

A índole essencialmente empírica ou teórica, a consideração ou não das energias vibracionais das moléculas reagentes e do complexo activado no zero absoluto e a distribuição estatística das energias em função da temperatura são os factores que diferenciam as definições das várias energias de activação.

A Cinética Química é um domínio científico em contínuo e rápido desenvolvimento. Ainda recentemente foi divulgado o papel relevante da Energia de Activação na relação estrutura molecular - reactividade quimica ${ }^{2}$,

O presente trabalho pretende mostrar a evolução do significado e da interpretação do termo genérico Energia de Activação.

\section{Energias de Activação}

\section{Altura da Barreira de Energia Potencial $-\mathrm{V}_{\mathrm{B}}$}

Se se considerarem os níveis energéticos correspondentes às mais estáveis configurações do complexo activado e dos reagentes, tendo em conta que o estado de transição se encontra no ponto mais elevado do percurso de energia mínima que transforma reagentes em produtos da reacção, a sua diferença representa o valor de $\mathrm{V}_{\mathrm{B}}-$ Altura da Barreira de Energia Potencial.

Deste modo foram excluídas na definição de $\mathrm{V}_{\mathrm{B}}$ as energias vibracionais, presentes mesmo no zero absoluto $\left(\Sigma_{/ \mathrm{n}} \mathrm{h} \nu_{\mathrm{n}} / 2\right)$, assim como outras, a temperaturas superiores.

$\mathrm{V}_{\mathrm{B}}$ é obtido por cálculos quânticos.

\section{Energia de Activação do Ponto Zero $-\Delta^{\ddagger} \mathrm{U}_{0}$}

De acordo com a equação fundamental da Teoria do Estado de Transição:

$$
\mathrm{k}=\frac{\mathrm{k}_{\mathrm{B}} \mathrm{T}}{\mathrm{h}} \chi \frac{\mathrm{Q}^{*}}{\mathrm{Q}_{\mathrm{r}}} \mathrm{e}^{-\Delta^{*} \mathrm{U}_{0} / \mathrm{RT}}
$$

onde $\mathrm{k}$ é a constante de velocidadé ou velocidade específica, $\mathrm{k}_{\mathrm{B}}$, h e $\mathrm{R}$, as constantes de Boltzmann, Planck e dos gases, respectivamente, $\chi$, o coeficiente de transmissão e $Q^{*}$ e $Q_{r}$, as funçð̃es de partição do complexo activado e dos reagentes, $\Delta^{*} \mathrm{U}_{0}-$ Energia (Interna) de Activação do Ponto Zero representa a diferença na energia potencial adicionada da energia do ponto zero, de carácter vibracional uma vez que energias translacionais e rotacionais são nulas a $0 \mathrm{~K}$, do complexo activado em relação aos reagentes.

Consequentemente, $\Delta^{*} U_{0}$ deve ser designado por Altura da Barreira corrigida para as Energias do Ponto Zero.

\section{Energia Interna de Activação $-\Delta^{*} \mathrm{U}_{\mathrm{T}}$}

Admitindo uma distribuição energética de Boltzmann com a temperatura:

$$
\mathrm{U}_{\mathrm{T}}^{\mathrm{o}}=\mathrm{U}_{0}^{\mathrm{o}}+\int_{0}^{\mathrm{T}} \mathrm{C}_{\mathrm{V}} \mathrm{dT}
$$

onde $\mathrm{U}_{\mathrm{T}}^{\circ}$ é a energia interna à temperatura $\mathrm{T}$ e $\mathrm{C}_{\mathrm{v}}$ é a capacidade calorífica a volume constante, quer para os reagentes quer para o complexo activado, tendo sido excluído, para esta última espécie, o grau de liberdade translacional correspondente ao movimento ao longo da coordenada reaccional, a diferença entre $U_{T}^{\circ}$ para o complexo activado e para os reagentes representa $\Delta^{*} \mathrm{U}_{\mathrm{T}}$ - Energia Interna de Activação à temperatura $\mathrm{T}$.

\section{Entalpia de Activação $-\Delta^{*} \mathrm{H}_{\mathrm{T}}$}

A entalpia relaciona-se com a energia interna pela conhecida expressão termodinâmica:

$$
\mathrm{H}=\mathrm{U}+\mathrm{pV}
$$

Consequentemente, a obtenção de $\Delta^{*} \mathrm{H}_{\mathrm{T}}-$ Entalpia de Activação pode fazer-se adicionando $\Delta(\mathrm{pV})$, diferença entre valores para o complexo activado e para os reagentes, a $\Delta^{\star} U_{\mathrm{T}}$.

Em reacções em fase gasosa, em condições ideais:

(i) unîmoleculares: $\Delta^{\star} \mathrm{H}_{\mathrm{T}}=\Delta^{*} \mathrm{U}_{\mathrm{T}}$

(ii) bimoleculares: $\dot{\Delta}^{*} \mathrm{H}_{\mathrm{T}}=\Delta^{*} \mathrm{U}_{\mathrm{T}}-\mathrm{RT}$

(iii) termoleculares: $\Delta^{\neq} \mathrm{H}_{\mathrm{T}}=\Delta^{\neq} \mathrm{U}_{\mathrm{T}}-2 \mathrm{RT}$

Em reacções em fase condensada, pV é desprezável e $\Delta^{*} \mathrm{H}_{\mathrm{T}}=\Delta^{\neq} \mathrm{U}_{\mathrm{T}}$.

\footnotetext{
a CECUL - Instituto Bento da Rocha Cabral, 14, 1200 Lisboa.
} 


\subsection{Energia de Activação de Arrhenius $-\mathrm{E}_{\mathrm{A}}$}

De todas as energias de activação mencionadas, a de uso mais comum é $\mathrm{E}_{\mathrm{A}}$ - Energia de Activação de Arrhenius, a qual, por esse facto, nos merece referência especial.

$\mathrm{E}_{\mathrm{A}}$ é uma quantidade empírica definida através da equação de Arrhenius:

$$
k=A \exp \left(-E_{A} / R T\right)
$$

onde A é a designado por "factor pré-exponencial". $\mathrm{E}_{\mathrm{A}}$ são ambas independentes da temperatura e determinam-se através do gráfico de variação de $1 \mathrm{n} \mathrm{k}$ com $1 / \mathrm{T}$.

A sua introdução na Cinética Química, tal como o nome indica, deve-se a Arrhenius, em $1889^{3}$. Este investigador, basicamente um electroquímico, no sentido de credibilizar a sua Teoria da Dissociação Electrolítica, efectuou estudos cinéticos, incluindo efeitos salinos, de reacçōes que envolviam, na maioria, electrólitos ${ }^{4}$. Deve-se, ainda, àquele investigador a ideia fundamentada da substituição do termo "constante de velocidade" por "velocidade específica", por ser $\mathrm{k}$ sensível a um grande número de variáveis, controvérsia que se veio a estender até aos dias de hoje. Arrhenius verificou que as amplitudes dos efeitos de temperatura na velocidade das reacçð̄es eram demasiado grandes para poderem ser interpretadas com base na variação das energias moleculares translacionais ou da viscosidade do meio com a temperatura. Explicou, então, o fenómeno admitindo a existência de moléculas reagentes de dois tipos, "activas" e "passivas", em equilíbrio, o qual era descrito pela equação de van't Hoff. A quantidade das primeiras aumentaria rapidamente com a temperatura, embora o seu número fosse sempre muito inferior ao das segundas. $\mathrm{k}$ seria proporcional à fracção das moléculas reagentes na forma "activa", sendo $\mathrm{E}_{\mathrm{A}}$ interpretado como a diferença de entalpia entre aqueles dois tipos de moléculas reagentes.

E curioso notar que, enquanto as moléculas "passivas" determinavam, em geral, as propriedades físicas e químicas do sistema, as moléculas "activas" determinavam as propriedades reaccionais.

Estudos anteriores de Hood tinham já sugerido a linearidade de $(\mathrm{d} 1 \mathrm{nk} / \mathrm{dT})_{\mathrm{p}}$ com $\mathrm{T}^{-2}$ e posteriores de Bodenstein verificaram ser logk função linear de $\mathrm{T}^{-14}$. Apesar disso, só cerca de quinze anos após a sua formulação a equação de Arrhenius começou a ser aceite. $\mathrm{O}$ desenvolvimento independente da Teoria das Colisões, nos anos vinte, não foi alheio a este processo, uma vez que a sua equação fundamental envolve uma relação k-T análoga à proposta empiricamente por Arrhenius. Por outro lado, o conceito de Arrhenius de "estado activado" das moléculas foi o percursor das modernas teorias das velocidades das reaç̧̃es, a Teoria do Estado de Transição, em particular. De facto, a formulação desta teoria requer a criação de um "complexo activado" no estado de transição, e a existência de um equilíbrio entre as moléculas dos estados inicial e de transição.

$\mathrm{O}$ avanço tecnológico, que permitiu uma maior precisão nos valores de $\mathrm{k}$, mostrou nas últimas décadas a não generalidade da equação de Arrhenius. No entanto, mesmo quando a sua aplicabilidade é duvidosa, o seu uso é ainda muito vasto: sobre resultados de fraca precisão, em pequenos intervalos de temperatura e com o intuito de obter valores interpolados, para além, evidentemente, do interesse pedagógico-didáctico da sua introdução na história da Cinética Química.

Têm sido sugeridas algumas razôes para justificar a não adaptação da equação de Arrhenius a resultados experimentais, nomeadamente, a presença de catalisadores e de mecanismos concorrentes, a acção relevante de solventes em reacções em solução, o controlo cinético por difusão, o efeito de túnel em reacçðes de transferência protónica, etc. Desenvolvimentos da equação de Arrhenius, principalmente no sentido da sua aplicação a intervalos de temperatura alargados têm sido propostos, como seja admitir A proporcional a uma potência $\mathrm{m}$ de $\mathrm{T}$ :

$$
\mathrm{k}=\mathrm{A}^{\prime} \mathrm{T}^{\mathrm{m}} \exp \left(-\mathrm{E}_{\mathrm{A}} / \mathrm{RT}\right)
$$

onde o parâmetro $A^{\prime}$ é independente da temperatura. Em reacçð̋es bimoleculares em fase gasosa, se se considerar, por hipótese, que as velocidades relativas das moléculas reagentes têm uma distribuição de Maxwell-Boltzmann, a energia de activação de Arrhenius é a diferença entre duas quantidades estatísticas, a energia média das colisø̃es eficazes e a energia média de todas as moléculas, que de um modo geral é dependente da temperatura ${ }^{4-6-7}$.

Neste caso,

$$
E_{A}=\Delta^{\neq} U_{T}+R\left\{T-\left[\frac{d \ln \chi}{d(1 / T)}\right]{ }_{p}\right\}
$$

e, se $\chi$ for unitário ou, pelo menos, independente da temperatura, a equação anterior reduz-se a:

$$
\mathrm{E}_{\mathrm{A}}=\Delta^{*} \mathrm{U}_{\mathrm{T}}+\mathrm{RT}
$$

A evolução do significado do termo Energia de Activação justifica, a nosso ver, a introdução em Cinética da Energia de Activação Experimental $-\mathrm{E}_{\text {exp }}$, já definida por Laidler ${ }^{5}$ :

$$
E_{\exp }=-R\left[\frac{d 1 n k}{d(1 / T)}\right]
$$

e para a qual não é imposta uma variação particular com a temperatura.

Na Tabela 1 apresentam-se algumas das equaçð̃es utilizadas para descrever a dependência da velocidade específica da temperatura ${ }^{8}$, assim como a relação de $\mathrm{E}_{\exp }$ com os parâmetros naquelas incluídos. A sua determinação é usualmente feita por um tratamento analítico que recorre ao método dos mínimos quadrados. Como se pode ver $\mathrm{n}$ referida tabela, apenas em condiçð̃es particulares $\mathrm{E}_{\exp } \equiv \mathrm{E}_{\mathrm{A}}$.

\begin{tabular}{ll} 
1nk $=f(T)$ & $E_{\text {exp }}$ \\
\hline $1 n k=A+B / T$ & $-R B\left(\equiv E_{A}\right)$ \\
$1 n k=A+B / T+C 1 n T$ & $-R(B+C T)$ \\
$1 n k=A+C 1 n T+D T$ & $-R /\left(C T+D^{2}\right)$ \\
$1 n k=A+B / T+C \ln +D T$ & $-R\left(B+C T+D^{2}\right)$ \\
$1 n k=A+B T^{C}$ & $R B C T^{C+1}$
\end{tabular}

Tabela 1 .

Variação da velocidade específica com a temperatura; $E_{\exp }$ 
A introdução de $\mathrm{E}_{\text {exp }}$ permite evitar ambiguidades na definição de $\mathrm{E}_{\mathrm{A}}$, utilizando-se a designação Energia de Activação de Arrhenius exclusivamente para uma quantidade termodinâmica invariável com $\mathrm{T}$.

$\mathrm{A}$ interpretação de $\mathrm{E}_{\mathrm{A}}$ segundo Arrhenius só tem sentido para processos elementares. Um exemplo característico é o da reacção:

$$
2 \mathrm{NO}+\mathrm{Cl}_{2} \rightarrow 2 \mathrm{ONCl}
$$

em fase gasosa, que apresenta para $\mathrm{E}_{\mathrm{A}}$ um valor negativo, o que seria inconcebível admitindo-a de passo único ${ }^{9}$. O mecanismo proposto para esta reacção assume a seguinte forma:

$$
\begin{aligned}
& \mathrm{NO}+\mathrm{Cl}_{2} \underset{\mathrm{k}_{-1}}{\stackrel{\mathrm{k}_{1}}{\rightleftarrows}} \mathrm{ONCl}_{2} \\
& \mathrm{ONCl}_{2}+\mathrm{NO} \stackrel{\mathrm{k}_{2}}{\longrightarrow} 2 \mathrm{ONCl}
\end{aligned}
$$

A aplicação do Princípio do Estado Estacionário ao intermediário $\mathrm{ONCl}_{2}$ e na situação extrema de $\mathrm{k}_{-1} \gg \mathrm{k}_{2}$, a reacção mostra-se de $3 .^{a}$ ordem. Considerando variação linear de $1 \mathrm{n} \mathrm{k}$ com $\mathrm{T}^{-1}$ para todas as reacções parcelares, obtém-se:

$$
E_{A}=-R\left[\frac{d \ln \left(k_{1} k_{2} / k_{-1}\right)}{d(1 / T)}\right]_{p}=E_{1}+E_{2}-E_{-1}
$$

onde $E_{1}, E_{2}$ e $E_{-1}$ são as energias de tipo arrheniano envolvidas nos passos a que correspondem, respectivamente, $\mathrm{k}_{1}, \mathrm{k}_{2}$ e $\mathrm{k}_{-1}$.

$\mathrm{O}$ valor negativo de $\mathrm{E}_{\mathrm{A}}$ pode, então, ser facilmente explicado:

$$
\mathrm{E}_{-1}>\mathrm{E}_{1}+\mathrm{E}_{2}
$$

Outros exemplos são possíveis, reacções em cadeia, de catálise heterogénea e de recombinação atómica, para as quais $\mathrm{E}_{\mathrm{A}}$ apresenta expressð̃es complexas em termos das energias dos processos elementares.

\section{Exemplo}

A reacção em fase gasosa

$$
\mathrm{D}+\mathrm{H}_{2} \rightarrow \mathrm{DH}+\mathrm{H}
$$

foi estudada experimental e teoricamente. Na Figura 1 apresenta-se uma comparação entre as diferentes energias de activação, atrás definidas, para esta reacção *. A esquerda representaram-se níveis energéticos respeitantes aos reagentes e, à direita, respeitantes ao complexo activado.

\section{Comentário final}

$\mathrm{Na}$ Cinética Química é possível discernir entre duas linhas de desenvolvimento: uma, de indole essencialmente empírica e, outra, teórica ${ }^{10}$. A lei de velocidade de uma reacção bem como a equação de variação da velocidade específica com a temperatura formulada por Arrhenius, e consequentemente $\mathrm{E}_{\mathrm{A}}$, são de carácter empírico, isto é, de verificação estritamente experimental.

Por outro lado, as equações resultantes da Teoria das Colisð̋es e da Teoria do Estado de Transição inserem-se na segunda linha de desenvolvimento. Assim,
$\Delta^{*} \mathrm{U}_{0}, \Delta^{\ddagger} \mathrm{U}_{\mathrm{T}}$ e $\Delta^{\neq} \mathrm{H}_{\mathrm{T}}$ são de carácter teórico.

Conceitos básicos, como os generalizados sob a designação de Energia de Activação, têm definições precisas e, embora muitas vezes interrelacionados, são numericamente distintos.

Em resumo, para uma reacção elementar podem estabelecer-se as seguintes igualdades:

$$
\begin{aligned}
& \Delta^{\ddagger} \mathrm{U}_{0}=\mathrm{V}_{\mathrm{B}}+\Delta\left(\sum_{\mathrm{n}} \mathrm{h} \nu_{\mathrm{n}} / 2\right) \\
& \Delta^{\neq} \mathrm{U}_{\mathrm{T}}=\Delta^{\neq} \mathrm{U}_{0}+\Delta\left(\int_{0}^{\mathrm{T}} \mathrm{C}_{\mathrm{V}} \mathrm{dT}\right) \\
& \Delta^{\neq} \mathrm{H}_{\mathrm{T}}=\Delta^{\neq} \mathrm{U}_{\mathrm{T}}+\Delta(\mathrm{pV}) \\
& \mathrm{E}_{\mathrm{A}}=\Delta^{\neq} \mathrm{U}_{\mathrm{T}}+\mathrm{R}\left\{\mathrm{T}-\left[\frac{\mathrm{d} 1 \mathrm{n} \chi}{\mathrm{d}(1 / \mathrm{T})}\right]_{\mathrm{p}}\right\}^{*}
\end{aligned}
$$

* $\mathrm{E}_{\mathrm{A}}$ segundo Logan 4 .

onde as variaçðes $\Delta$ representam as diferenças entre as funçð̃es expressas para o complexo activado e para os reagentes.

É frequente a apresentação, em livros de texto elementares, de diagramas de energia em função de uma coordenada reaccional em que $\mathrm{E}_{\mathrm{A}} \equiv{ }^{7} \mathrm{U}_{0} \equiv \overrightarrow{\mathrm{V}}_{\mathrm{B}}$. Esta simplificação pode conduzir a incorrecções na interpretação daqueles conceitos. O problema ainda se torna mais complicado quando face a reacções extremamente rápidas e de mecanismo complexo.

\section{Referências}

1. P.D. Pacey, J. Chem. Educ., 1981, 58, 612.

2. S.J. Formosinho, Rev. Port. Quím., 1985, 27, 427.

3. S. Arrhenius, Z. Physik. Chem., 1889, 4, 226.

4. S.R. Logan, J. Chem. Educ., 1982, 59, 279.

5. K.J. Laidler, J. Chem. Educ., 1984, 61, 494.

6. D.G. Truhlar, J. Chem. Educ., 1978, 55, 309.

7. S.J. Formosinho, Rev. Port. Quim., 1975, 17, 268.

8. L. Albuquerque e R. Gonçalves, "Cinética Química", Escolar Ed., Lisboa, 1986.

9. S.R. Logan, Educ. in Chem., 1986, 23, 148.

10. S.R. Logan, Educ. in Chem., 1984, 21, 20.

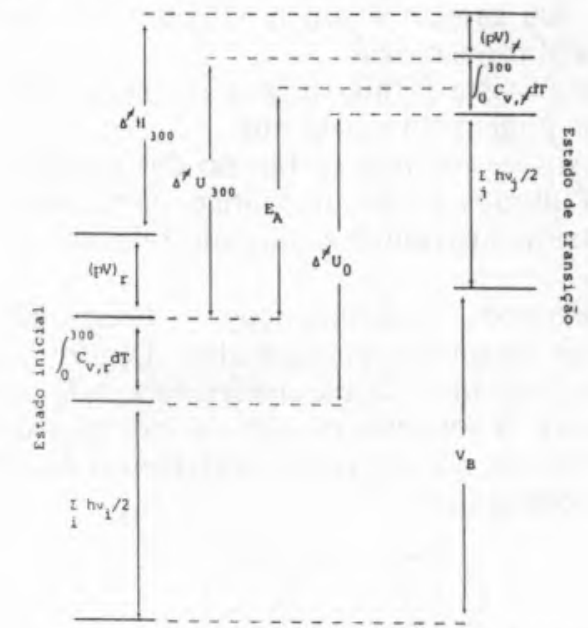

$$
\begin{aligned}
& V_{B}=40,50 \mathrm{~kJ} \mathrm{~mol}^{-1} \\
& \triangle F_{U_{0}}=35,6 \\
& \mathrm{E}_{\mathrm{A}}=27,40 \\
& *_{0}=32,2 \\
& { }^{7} \mathrm{H}_{\mathrm{Fr}}=29,7
\end{aligned}
$$

$$
\begin{aligned}
& \mathrm{Chv}_{\mathrm{L}} / 2=25,9 \mathrm{~kJ} \mathrm{~mol}^{-1} \\
& \mathrm{r}_{\mathrm{hv}} / 2=20,9 \\
& \int_{0}^{m} c_{v, r} c^{d n}=10,0 \\
& \int_{0}^{0 m} c_{v, f r} c_{v}=6,7 \\
& (p v)_{x}=5,0 \\
& (p v)_{x}=2,5 \text {. }
\end{aligned}
$$

Figura 1 .

Esquema energético para a reacção: $\mathrm{D}+\mathrm{H}_{2} \rightarrow \mathrm{HD}+\mathrm{H}$

\footnotetext{
${ }^{*} \mathrm{E}_{\mathrm{A}}$ segundo Logan ${ }^{4}$.
} 